\title{
MODELACION DE FLUJO 1D EN EL RIO UCAYALI
}

\author{
Victor A. Yzocupe \\ Laboratorio de Fluidodinámica Computacional, Departamento de Ingeniería Mecánica de Fluidos, \\ Facultad de Ciencias Físicas, Universidad Nacional Mayor de San Marcos. Lima, Perú
}

\begin{abstract}
Resumen
Se presenta la aplicación de un modelo numérico unidimensional para la simulación de flujo en el río Ucayali. El modelo es general y flexible; porque permite simular un amplio rango de condiciones de flujo, tales como flujo uniforme y no uniforme, estacionario y transitorio. El modelo no considera problemas de filtración, transporte de sedimentos ni viento.

El modelo se basa en la aproximación numérica de las ecuaciones gobernantes del flujo en canales abiertos (ecuaciones de Saint Venant) mediante el esquema de diferencias finitas implícito de Preissmann. Las variables dependientes son el caudal (Q) y el nivel de la superficie del agua (Z). Los algoritmos aplicados en los segmentos del canal con las condiciones de frontera componen un sistema de ecuaciones lineales, el mismo que se resuelve mediante el método de Doble Barrido.

La aplicación se ha realizado a un tramo de $22 \mathrm{kms}$ del río Ucayali, cerca de la ciudad de Pucallpa. Se presentan dos pruebas, una para flujo estacionario y otra para flujo no estacionario, las mismas que demuestran el buen desempeño del modelo presentado.
\end{abstract}

Palabras claves: Modelo numérico, hidráulica de ríos, hidráulica computacional, flujo unidimensional.

\begin{abstract}
It presents the formulation of a one-dimensional numerical model for simulation of flow in the Ucayali river. The model is general and flexible; because it permits to simulate a broad range of flow conditions, such as uniform and non-uniform flow, steady and unsteady. The model does not consider problems of filtration, sediment transport, neither wind.

The model is based in the numerical approximation of the governing equations of flow in open channels (Saint Venant's equations) using the implicit finite difference Preissmann scheme. The dependent variables are the discharge $(\mathrm{Q})$ and the water surface level $(\mathrm{Z})$. The algorithms applied in the segments of the channel plus the boundary conditions compose one system of lineal equations, which is solved using the Double Sweep method.

The application is done to a reach of $22 \mathrm{kms}$ of the Ucayali River, near to the Pucallpa city. It presents two tests, one for steady flow and another for non-steady flow, which demonstrate the good performance of the model.
\end{abstract}

Keywords: Numerical model, hydraulics of rivers, computational hydraulics, one-dimensional flow.

\section{Introducción}

El modelamiento de flujo en ríos y canales es una potente herramienta para proyectos de investigación y de ingeniería, en los que se necesitan conocer la distribución de caudales o niveles a lo largo de un sistema fluvial o el transporte de sedimentos.

En hidráulica fluvial, existen muchos casos donde los parámetros del flujo varían con el tiempo. Un ejemplo es el desplazamiento de ondas de crecida en ríos; una onda de crecida es el aumento lento y gradual del nivel de la superficie en un tramo de canal. Otro caso, es el desplazamiento de la onda generada por el rompimiento de una presa [8]. 
Si tenemos un tramo de canal artificial con muy baja resistencia y una onda de crecida moviéndose a través de éste; entonces, la configuración de la onda permanecerá casi inalterada. Sin embargo, en un cauce real, la resistencia es alta debido a su irregularidad; por tanto, la configuración de la onda se modifica continuamente a lo largo de su recorrido. La determinación de esta modificación se conoce como desplazamiento de crecidas y se puede aplicar tanto a canales como a embalses [4].

El desplazamiento de crecidas es útil para la implementación de un programa de control de crecidas y para la operación de un servicio de predicción de inundaciones. El desplazamiento de crecidas se conoce como un procedimiento que simula el proceso y produce un registro continuo en las secciones de control elegidas sobre el curso de agua, teniendo como punto de partida un hidrograma de crecidas en la frontera aguas arriba.

Los términos "canal", "tramo" o "ramal" se usan indistintamente para definir una cierta longitud de canal, con características geométricas o hidráulicas homogéneas, o que esta controlado por alguna condición de frontera externa o interna. La subdivisión de un canal o tramo se conoce como un "segmento".

\section{Formulación del Modelo Numérico}

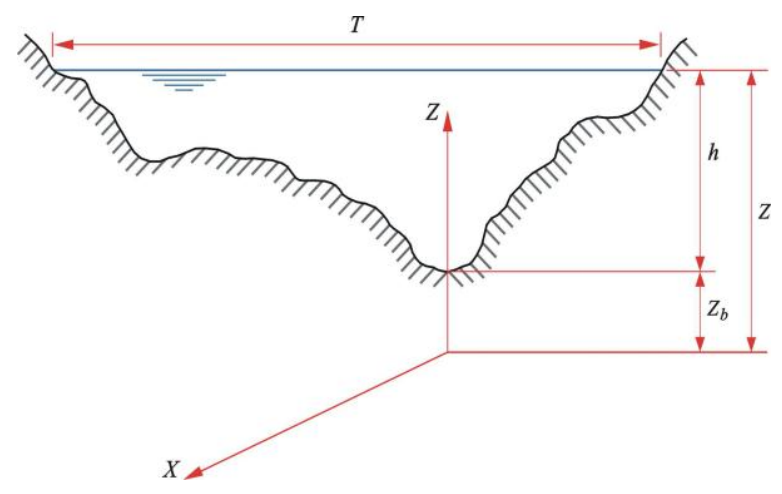

Fig. 1. Sistema de Coordenadas

Las ecuaciones que describen el flujo no estacionario unidimensional en canales abiertos son la ecuación de continuidad y la ecuación de movimiento. El desarrollo de la ecuación de continuidad está basado en el principio de conservación de la masa, mientras que la ecuación de movimiento se puede desarrollar a partir de la segunda Ley de Newton o de la conservación de la cantidad de movimiento.

Las ecuaciones gobernantes del flujo en canales se sustentan en un sistema de coordenadas $X-Z$, cuyo origen vertical se puede ubicar en el nivel de la frontera aguas abajo o en forma más general en el nivel medio del mar.

El eje $X$ se utiliza para representar la distancia longitudinal del canal y es positivo hacia aguas abajo del canal. El eje $Z$ representa el nivel de la superficie líquida, y $Z_{b}$ es el nivel del fondo del canal, $h$ representa la altura neta de la columna de agua.

\subsection{Ecuaciones Gobernantes}

El método hidráulico se basa en la solución de las ecuaciones diferenciales parciales de conservación de masa y de conservación de la cantidad de movimiento [8]. Estas ecuaciones también se conocen como las Ecuaciones de Aguas Rasas o de Saint Venant. La descarga $(Q)$ y el nivel de la superficie del agua $(Z)$ son las variables dependientes.

Ecuación de Conservación de Masa [6]:

$$
T \frac{\partial Z}{\partial t}+\frac{\partial Q}{\partial x}=0
$$

Ecuación de Conservación de Cantidad de Movimiento [6]:

$$
\frac{\partial Q}{\partial t}+\frac{\partial}{\partial x}\left(\beta \frac{Q^{2}}{A}\right)+g A \frac{\partial Z}{\partial x}+g A S_{f}=0
$$

donde $\beta$ y $S_{\mathrm{f}}$ se definen como [3]:

$$
\begin{gathered}
\beta=\frac{1}{U^{2} A} \int_{0}^{T} u^{2} d A ; \quad \beta=\beta(Z) \\
S_{f}=\frac{n^{2} Q^{2}}{A^{2} R^{4 / 3}}
\end{gathered}
$$

y el término no lineal de la ecuación (2) se descompone así [3]:

$$
\frac{\partial}{\partial x}\left(\beta \frac{Q^{2}}{A}\right)=2 \beta \frac{Q}{A} \frac{\partial Q}{\partial x}-\beta \frac{Q^{2}}{A^{2}} \frac{\partial A}{\partial x}
$$




$$
\begin{gathered}
\frac{\partial A}{\partial x}=\frac{\partial A}{\partial Z} \frac{\partial Z}{\partial x}+\left.\frac{\partial A}{\partial x}\right|_{Z=c t e}=T \frac{\partial Z}{\partial x}+\left.\frac{\partial A}{\partial x}\right|_{Z=c t e} \\
T=T(Z), \quad A=A(Z)
\end{gathered}
$$

Reemplazando todas las anteriores ecuaciones en (2) obtenemos:

$$
\begin{aligned}
& \frac{\partial Q}{\partial t}+2 \beta \frac{Q}{A} \frac{\partial Q}{\partial x}+\left(g A-\beta \frac{Q^{2}}{A^{2}} T\right) \frac{\partial Z}{\partial x} \\
& -\left.\beta \frac{Q^{2}}{A^{2}} \frac{\partial A}{\partial x}\right|_{Z=c t e}+g \frac{n^{2}|Q| Q}{A R^{4 / 3}}=0
\end{aligned}
$$

En estas ecuaciones, la distancia longitudinal a lo largo del thalweg del canal $(x)$, y el tiempo transcurrido $(t)$, son las variables independientes (la distancia longitudinal $(x)$, y el caudal o descarga $(Q)$, son positivas en la dirección aguas abajo). Las otras cantidades se definen como:

$Z$ nivel de la superficie del agua, referida al nivel medio del mar $=Z_{b}+h$,

$Z_{\mathrm{b}}$ nivel del lecho, referida al nivel medio del mar,

$h$ profundidad vertical del flujo o altura de lámina de agua,

$S_{\mathrm{f}}$ pendiente de fricción,

$\beta$ coeficiente de distribución de velocidad o de Boussinesq,

$g$ aceleración gravitacional,

$A$ área mojada de la sección transversal,

$R$ radio hidráulico de la sección transversal,

$P$ perímetro mojado de la sección transversal,

$T$ ancho total de la superficie líquida, transversal a la dirección del flujo,

$u \quad$ velocidad del flujo en un punto de la sección transversal,

$U$ velocidad media del flujo $=Q / A$,

$n$ coeficiente de rugosidad de Manning.

El radio hidráulico $(R)$ se utiliza en la ecuación (3) y en todas las demás ecuaciones; pero, cuando el canal tiene una configuración geométrica arbitraria (canal natural), se puede sustituir el radio hidráulico por la profundidad hidráulica. Esta aproximación $(R \approx A / T)$ se asume válida para cuerpos de agua poco profundos [4], y se utiliza por la facilidad de calcular el ancho de la superficie líquida en lugar del perímetro hidráulico.
El coeficiente de Boussinesq, $\beta$, también llamado el coeficiente de momentum, está presente en la ecuación de movimiento para tomar en cuenta las distribuciones de velocidad no uniformes en las secciones transversales.

Las ecuaciones (1) y (6) describen, en general, el flujo no permanente en un canal de sección arbitraria teniendo áreas de transporte y de almacenamiento (o solamente de transporte). En su formulación, se asume que el agua es de densidad homogénea, que la presión hidrostática prevalece en todo el canal, que la pendiente de fondo del canal es pequeña y uniforme, que no hay procesos de transporte de sedimentos en el lecho del canal (no ocurre erosión ni sedimentación), que la geometría del tramo es suficientemente uniforme para permitir la aproximación unidimensional, y que la resistencia por fricción es la misma como en el flujo permanente, permitiendo el uso de la ecuación de Chézy o Manning.

\subsection{Esquema de Diferencias Finitas Implicito de Preissmann}

Existen numerosos métodos numéricos para producir soluciones aproximadas de las ecuaciones de flujo. En este trabajo, las ecuaciones de flujo serán discretizadas mediante el esquema de diferencias finitas implícito de Preissmann. Esta técnica, permite que el modelo utilice segmentos de diferentes longitudes y un esquema que va desde centrado hasta totalmente adelantado en el tiempo [2].

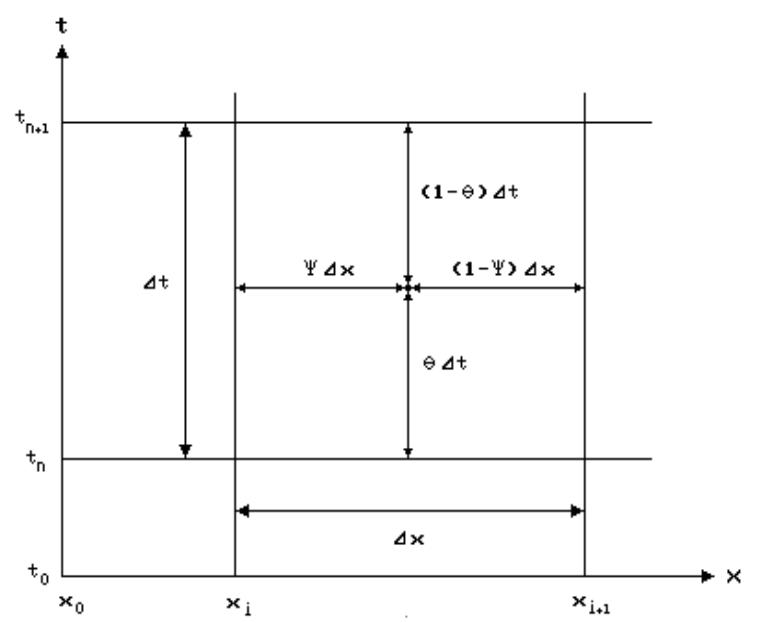

Fig. 2. Grilla espacio-temporal del Esquema Preissmann 
El método de solución implícito se emplea debido a su eficiencia inherente y propiedades de estabilidad superior. Es posible agregar un procedimiento de iteración opcional controlable por el usuario para mejorar la exactitud de los resultados.

El sistema de grilla espacio-temporal de la figura 2 muestra la región en que las ecuaciones de flujo son resueltas. Las derivadas temporal y espacial de la función, $f$, que representa la variable dependiente nivel o caudal, se discretizan de la siguiente manera [2]:

$$
\begin{gathered}
\frac{\partial f}{\partial t} \approx(1-\psi) \frac{f_{i}^{n+1}-f_{i}^{n}}{\Delta t}+\psi \frac{f_{i+1}^{n+1}+-f_{i+1}^{n}}{\Delta t} \\
\Delta t=\Delta t_{1}=\ldots=\Delta t_{n} \\
\frac{\partial f}{\partial x} \approx(1-\theta) \frac{f_{i+1}^{n}-f_{i}^{n}}{\Delta x_{i}}+\theta \frac{f_{i+1}^{n+1}-f_{i}^{n+1}}{\Delta x_{i}}
\end{gathered}
$$

Donde, $0 \leq \psi \leq 1$, y $0.5 \leq \theta \leq 1$ son factores de ponderación utilizados para especificar la posición temporal y espacial, respectivamente, dentro del incremento de tiempo $\Delta \mathrm{t}_{\mathrm{n}}$ e incremento de distancia $\Delta \mathrm{x}_{\mathrm{i}}$ en el cual la derivada y las funciones serán evaluadas.

Tomando $\psi=\frac{1}{2}$, produce una derivada temporal promedio en la posición espacial $i+\frac{1}{2}$, y similarmente, cuando $\theta=\frac{1}{2}$ la derivada espacial esta centrada en la dirección temporal $n+\frac{1}{2}$, los errores de truncamiento son del orden de $\Delta t^{2}$ y $\Delta x^{2}$ respectivamente. Pero tomando $\theta>\frac{1}{2}$ se introducen errores de truncamiento que producen disipación numérica. Las derivadas temporales normalmente son calculadas con $\psi=\frac{1}{2}$, aunque otros valores pueden ser ventajosos cuando se utilizan segmentos de longitudes desiguales [2].

De una manera similar al tratamiento de la derivada espacial, el área de la sección transversal, el ancho de la superficie libre, el radio hidráulico, y las descargas en forma no derivativa, denotadas por $f(x, t)$, se discretizan como sigue [5]:

$$
f(x, t) \approx(1-\chi) \frac{f_{i+1}^{n}+f_{i}^{n}}{2}+\chi \frac{f_{i+1}^{n+1}+f_{i}^{n+1}}{2}
$$

El factor de ponderación $\chi$ se encuentra en el rango $0 \leq \chi \leq 1$. Así, estos valores funcionales pueden ser representados en cualquier nivel de tiempo como las derivadas espaciales.

La determinación de valores apropiados para estos parámetros es importante porque ellos tienen efecto en la precisión, convergencia, y estabilidad del modelo. Tales valores son la determinación del incremento de tiempo $(\Delta \mathrm{t})$, la longitud de los segmentos del canal $(\Delta x)$, y la selección de los factores apropiados de ponderación del esquema Preissmann.

La solución numérica de las ecuaciones de flujo en un sistema de grilla rectangular x-t, impone una restricción en la determinación del incremento de tiempo basado en la longitud de los segmentos. Esta restricción es aplicable, en un sentido matemático riguroso, a la técnica de diferencias finitas explícita y se denomina Condición de Courant-Friedrichs-Lewy [1],

$$
\Delta t \leq \frac{\Delta x}{|U \pm \sqrt{g h}|}
$$

En esta relación, $\Delta t$ es el incremento de tiempo, $\Delta x$ es la longitud del segmento, $U$ es la velocidad media del flujo, $g$ es la aceleración de la gravedad y $h$ es la profundidad del flujo. Esta condición asegura condiciones de cálculo estable y requiere excesiva cantidad de tiempo de cómputo; por esta razón las técnicas de solución implícitas ofrecen ventajas económicas. No obstante, la Condición de Courant, la cual es una función de la celeridad de onda $(c=\sqrt{g h})$ y la velocidad del flujo, constituyen un valioso índice cuando seleccionamos el incremento de tiempo para una solución implícita.

\subsection{Discretización de las Ecuaciones Gobernantes}

Las ecuaciones diferenciales parciales de flujo (1) y (6) son transformadas en expresiones discretas mediante la aplicación del esquema de diferencias finitas implícito de Preissmann, utilizando los operadores definidos en las ecuaciones (7), (8) y (9). Se utiliza la tilde (־) para denotar las cantidades tomadas como constantes locales, las que se actualizan a través de las iteraciones en el proceso de cálculo. 
La ecuación de continuidad se reduce a [8]:

$$
a_{i} Z_{i}^{n+1}-Q_{i}^{n+1}+a_{i} Z_{i+1}^{n+1}+Q_{i+1}^{n+1}=b_{i}
$$

donde,

$$
\begin{aligned}
& a_{i}=\frac{\tilde{T}_{i} \Delta x_{i}}{2 \theta \Delta t} \\
& b_{i}=a_{i}\left(Z_{i}^{n}+Z_{i+1}^{n}\right)+\frac{(1-\theta)}{\theta}\left(Q_{i}^{n}-Q_{i+1}^{n}\right)
\end{aligned}
$$

y la ecuación de movimiento se reduce a [8]:

$$
-Z_{i}^{n+1}+c_{i} Q_{i}^{n+1}+Z_{i+1}^{n+1}+d_{i} Q_{i+1}^{n+1}=e_{i}
$$

donde,

$$
\begin{gathered}
c_{i}=f_{i}-p_{i}+h_{i} \quad d_{i}=f_{i}+p_{i}+h_{i} \\
f_{i}=\frac{\Delta x_{i}}{2 \theta g \tilde{A}_{i} \Delta t} \quad p_{i}=\frac{2 \beta \tilde{Q}_{i}}{g \tilde{A}_{i}^{2}} \\
h_{i}=\frac{\chi \Delta x_{i} n^{2}\left|\tilde{Q}_{i}\right|}{2 \theta \tilde{A}_{i}^{2} \tilde{R}_{i}^{4 / 3}} \\
e_{i}=\frac{(1-\theta)}{\theta}\left(Z_{i}^{n}-Z_{i+1}^{n}\right)+\left[f_{i}+\frac{(1-\theta)}{\theta} p_{i}-\frac{(1-\chi)}{\chi} h_{i}\right] Q_{i}^{n} \\
+\left[f_{i}-\frac{(1-\theta)}{\theta} p_{i}-\frac{(1-\chi)}{\chi} h_{i}\right] Q_{i+1}^{n} \\
+\frac{\beta \tilde{Q}_{i}^{2}}{\theta g \tilde{A}_{i}^{3}}\left[\tilde{T}_{i}\left(\tilde{Z}_{i+1}-\tilde{Z}_{i}\right)+\left.\left(\tilde{A}_{i+1}-\tilde{A}_{i}\right)\right|_{Z=c t e}\right]
\end{gathered}
$$

Para ii puntos de cálculo se generará un sistema de (2ii-2) ecuaciones algebraicas lineales con $2 i i$ incógnitas, por lo que se necesita adicionar dos ecuaciones, las que se toman de las condiciones de frontera, para completar el número de ecuaciones necesarias. Es decir, se obtiene un sistema matricial con una matriz de coeficientes pentadiagonal, $A$, de orden $2 i i$, un vector de incógnitas, $x$, de tamaño $2 i i$, y un vector de residuos, $b$, de tamaño $2 i i$.

El procedimiento de simulación se realiza para cada paso de tiempo; es decir, dadas las condiciones iniciales en el tiempo $t_{0}$, donde todos los valores de las variables se conocen $\left(Z_{i}^{n}, Q_{i}^{n}\right)$, procedemos al cálculo del paso de tiempo siguiente, $t_{1}$, en el cual, dada una condición de frontera aguas arriba procedemos al ensamblaje de las ecuaciones correspondientes en todos los puntos de grilla a lo largo del tramo en estudio. Luego, adicionamos la ecuación correspondiente a la condición de frontera aguas abajo. Así, completamos el sistema matricial de ecuaciones que se procede a resolver mediante el método de Doble Barrido [8].

La solución del sistema de ecuaciones proporciona los niveles y caudales en todos los puntos de la grilla, luego de lo cual se proporcionan los valores calculados a las variables del nivel de tiempo $n+1\left(Z_{i}^{n+1}, Q_{i}^{n+1}\right)$. (la primera aproximación en el proceso de iteración fue al adoptar los valores del tiempo precedente). Se pueden realizar iteraciones dentro de un mismo paso de tiempo para mejorar la precisión de la solución, pero con una segunda iteración se obtiene una solución satisfactoria. Esta iteración significa recalcular los coeficientes de las ecuaciones lineales [9].

Para el segundo paso de tiempo se procede de manera idéntica al proceso anterior; pero, previamente se transfiere la solución obtenida a las variables del nivel de tiempo $n\left(Z_{i}^{n}, Q_{i}^{n}\right)$. Este procedimiento se repite hasta completar todos los pasos de tiempo que requiere la simulación [9].

\section{Aplicación al Río Ucayali}

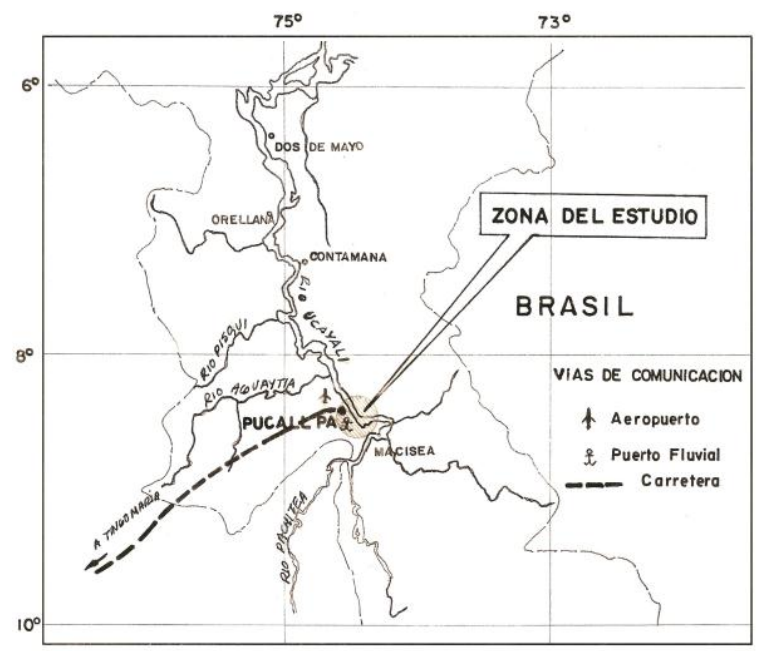

Fig. 3. Ambito geográfico del Río Ucayali

La aplicación del modelo se ha realizado al río Ucayali, el cual ha sido seleccionado por ser uno de los poquísimos ríos del país que cuentan con estudios hidráulicos detallados y con la información topográfica e hidrológica que se 
necesita para la aplicación del presente modelo. El tramo de estudio esta ubicado en el departamento de Ucayali, provincia de Pucallpa.

Para este trabajo se ha utilizado la información del "Estudio Hidráulico y Morfológico del Río Ucayali", realizado por la Dirección de Hidrografía y Navegación de la Marina de Guerra y por la Dirección General de Transporte Acuático del Ministerio de Transportes y Comunicaciones del Perú [8].

Específicamente, se han tomado los datos del informe final e informes trimestrales del año 1983 y del último informe trimestral de 1992. Se tomó el período del año 1983 por ser un año con mejorías en la toma y en el procesamiento de datos.

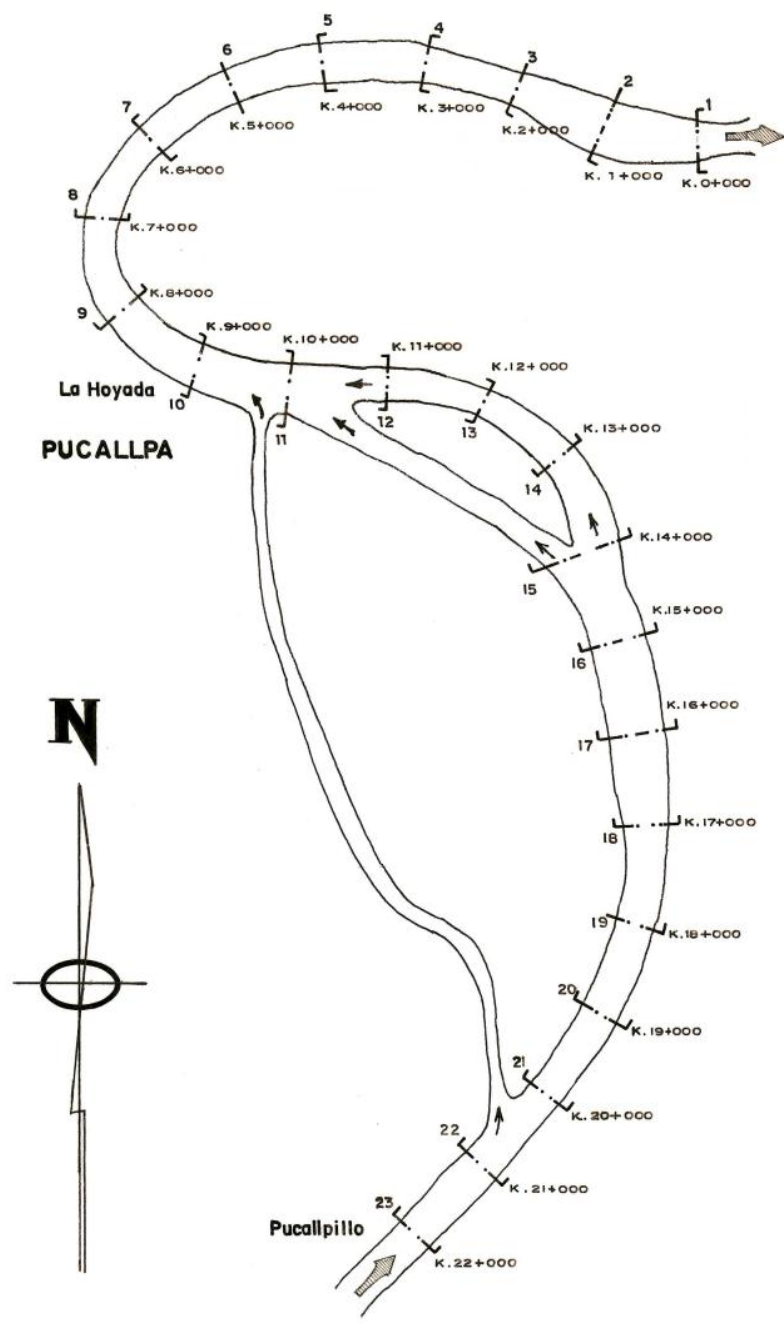

Fig. 4. Río Ucayali - tramo de Pucallpa. Secciones transversales y distancias longitudinales

\subsection{Topología del Sistema}

El siguiente paso consiste en definir la topología del sistema a ser simulado. Esto se ha visto facilitado por el esquema diseñado en el estudio existente. Se tiene un plano del área circundante de la Ciudad de Pucallpa a escala 1:20,000, donde ya existe un esquema definido. Se delimitó el estudio a un trayecto de $22 \mathrm{~km}$. divididos en 22 tramos de $1 \mathrm{~km}$. cada uno, lo que hace un total de 23 secciones transversales. Estas 23 secciones están numeradas en orden ascendente de aguas abajo hacia aguas arriba.

Existen sólo dos estaciones hidrométricas a lo largo del tramo de estudio. La Estación Pucallpillo en la sección 23 y la Estación La Hoyada en la sección 10. Se eligió a la Estación Pucallpillo como la frontera aguas arriba y la Sección 01 como la frontera aguas abajo del sistema modelado. De esta forma, la Estación La Hoyada será nuestro punto de comprobación, donde se contrastarán los registros históricos con los niveles calculados por el modelo.

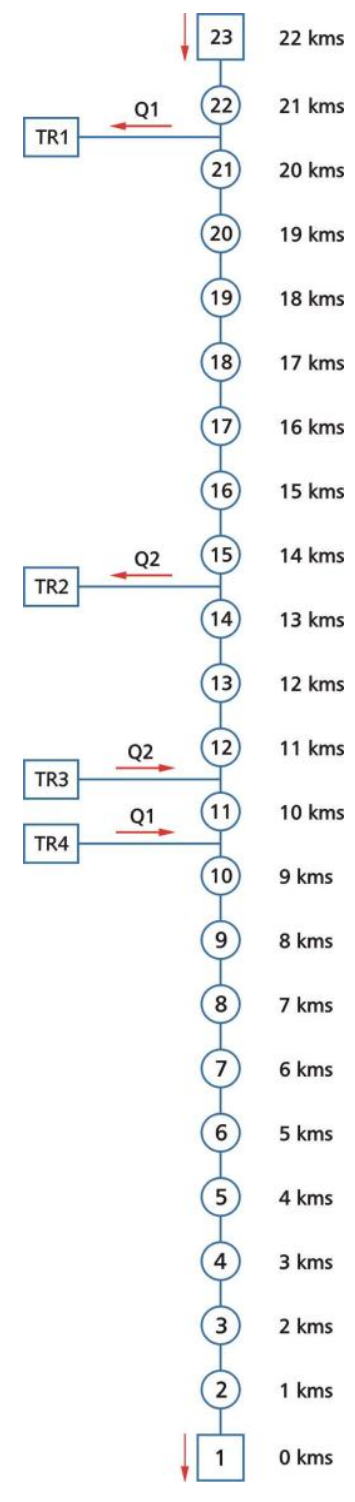

Fig. 5. Topología del tramo en estudio

\subsection{Datos Hidrológicos Utilizados}

La Estación Hidrométrica de Pucallpillo cuenta con registros diarios de niveles de la superficie líquida tomadas a las 08:00 hrs. La Estación Hidrométrica de La Hoyada también cuenta con registros diarios de niveles tomados a las 08:00 y 18:00 hrs. La Estación Pucallpillo cuenta además con la siguiente información: 
- Aforos semanales: mediciones de velocidad.

- Parámetros hidráulicos: medición del área, ancho de la superficie, cálculo de la pendiente de la superficie líquida.

- Curva de calibración caudal-nivel.

- Curva de calibración área-nivel.

- Curva de calibración velocidad-nivel.

- Curva de relación coeficiente de rugosidad de Chezy-nivel.

Todas las curvas de calibración están basadas en datos de los años 1982 y 1983 y cuentan con sus correspondientes ecuaciones de ajuste. De ellas la única que se ha utilizado es la curva de calibración caudal-nivel. Los coeficientes de Manning sirvieron para iniciar el proceso de calibración.

\subsection{Datos Topográficos Utilizados}

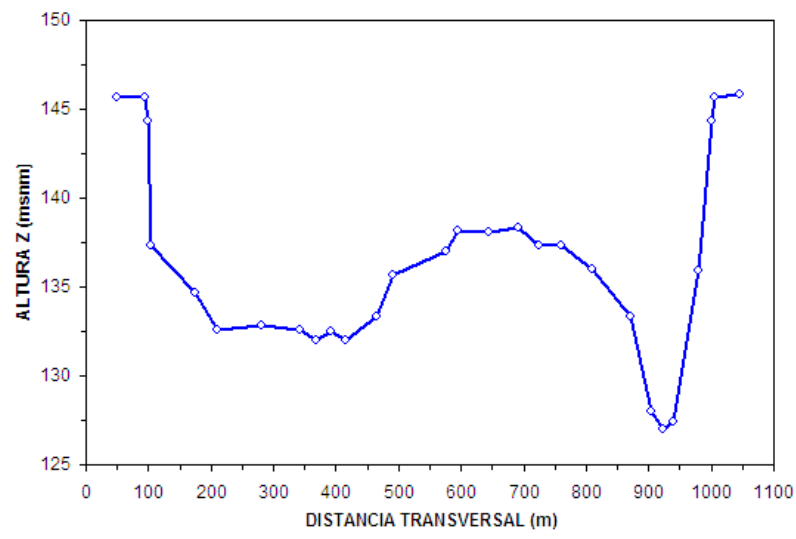

Fig. 6. Sección transversal 01. Distancia longitudinal 0 kms. Fecha medición: 05/03/83

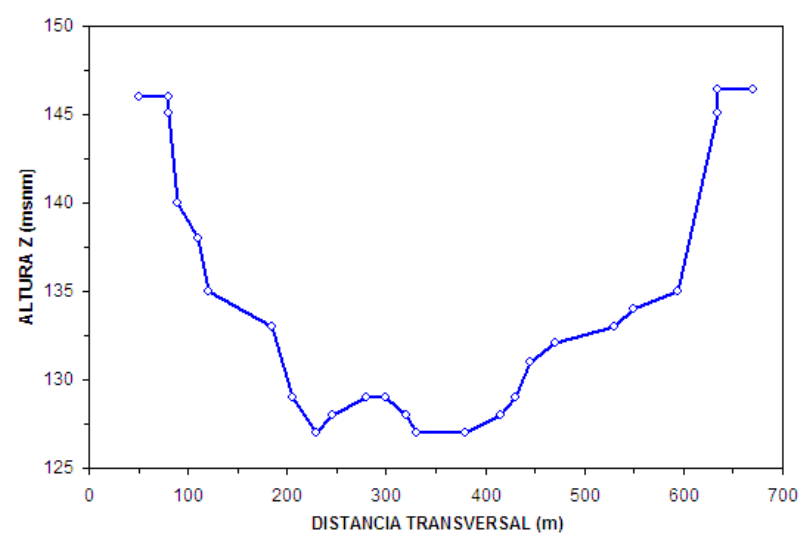

Fig. 7. Sección transversal 23. Distancia longitudinal 22 kms. Fecha medición: 21/02/83

Se cuenta con un mapa del área circundante de la Ciudad de Pucallpa a escala 1:20,000 y los planos batimétricos de todas las secciones transversales a escala horizontal 1:5,000 y vertical 1:100, medidos entre los meses de febrero, marzo y mayo de 1983.

\subsection{Generación de Parámetros Hidráulicos}

Los datos topográficos de cada una de las secciones transversales fueron digitalizados $\mathrm{y}$ procesados para obtener los siguientes parámetros hidráulicos; el área transversal, el ancho superficial y el perímetro mojado. Estos parámetros se calcularon para un rango de niveles definido entre el punto más bajo y la altura del margen más bajo de la sección transversal.
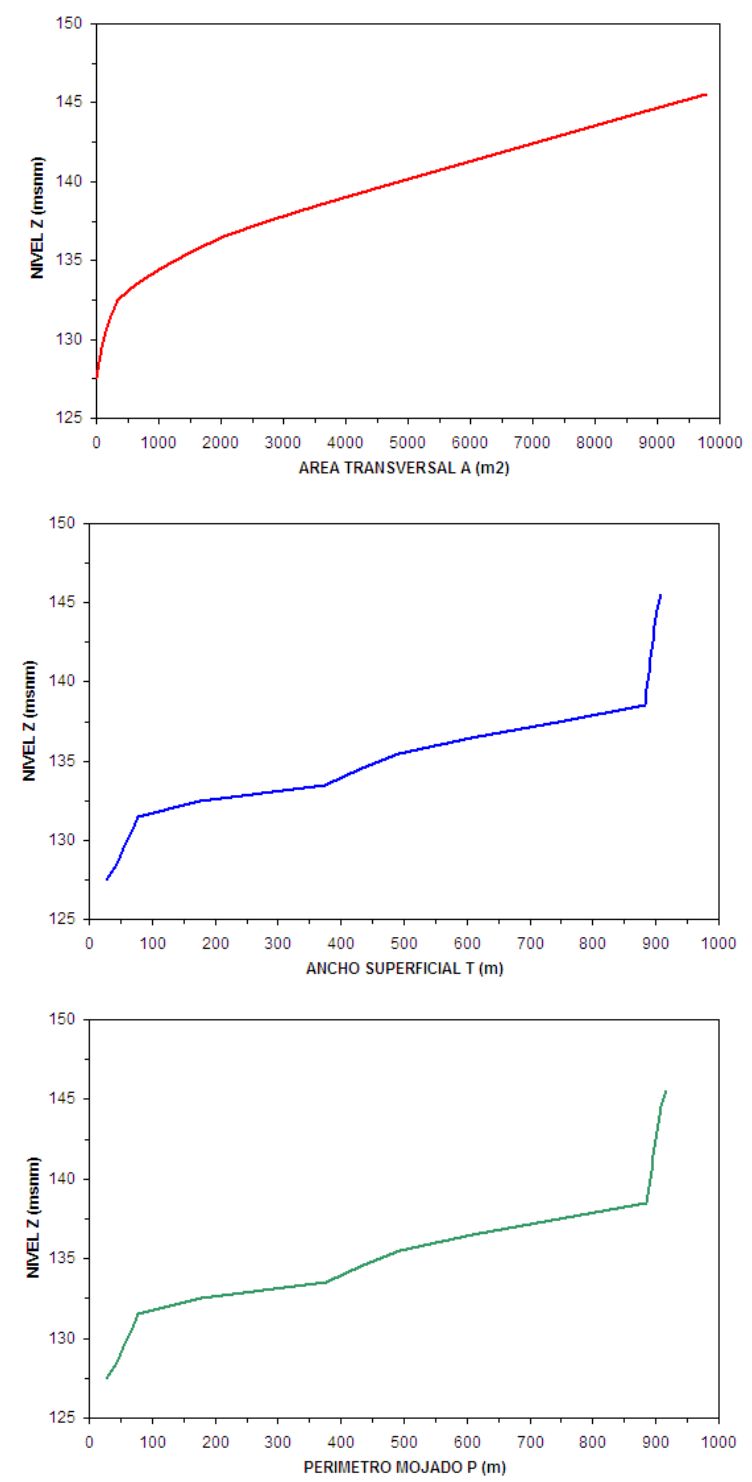

Fig. 8. Variación del área transversal, ancho superficial y perímetro mojado, según el nivel del agua, en la sección transversal 01 
Esta información sirve para que el modelo, mediante una interpolación lineal, calcule para cualquier nivel de agua dado, el área transversal, el ancho de la superficie líquida, el perímetro mojado o el radio hidráulico.

\subsection{Condiciones Iniciales}

Para iniciar la solución del sistema de ecuaciones, se requiere conocer los valores de las variables del flujo en el tiempo inicial. Estos valores se pueden obtener de datos medidos o calculados de alguna otra fuente, tales como aproximaciones para un estado permanente, o pueden ser los resultados de alguna simulación anterior.

Se tomaron como condiciones iniciales los niveles de la superficie liquida medidos en todas las secciones transversales. Como no se cuenta con esta información en un mismo momento para todas las secciones, se han tomado los niveles medidos entre los días $2,3,4$ y 5 de marzo de 1983, interpolándose en las secciones que no cuentan con este dato.

Tabla 1. Niveles de la Superficie y de Fondo

\begin{tabular}{|c|c|c|}
\hline SECCION & $\mathrm{Z}_{\mathrm{b}}(\mathrm{msnm})$ & $\mathrm{Z}(\mathrm{msnm})$ \\
\hline 1 & 127.0 & 144.33 \\
2 & 130.8 & 144.45 \\
3 & 129.85 & 144.45 \\
4 & 130.1 & 144.57 \\
5 & 123.8 & 144.57 \\
6 & 118.2 & 144.69 \\
7 & 118.0 & 144.69 \\
8 & 117.2 & 144.73 \\
9 & 127.8 & 144.77 \\
10 & 130.3 & 144.81 \\
11 & 128.6 & 144.85 \\
12 & 121.1 & 144.89 \\
13 & 129.3 & 144.95 \\
14 & 133.0 & 145.00 \\
15 & 134.8 & 145.02 \\
16 & 130.8 & 145.14 \\
17 & 123.3 & 145.14 \\
18 & 122.4 & 145.20 \\
19 & 123.2 & 145.27 \\
20 & 127.0 & 145.39 \\
21 & 128.7 & 145.39 \\
22 & 124.7 & 145.51 \\
23 & 127.0 & 145.55 \\
\hline
\end{tabular}

A partir de estos niveles el modelo calcula el caudal normal utilizando la ecuación de Manning. Estos dos valores constituyen las condiciones iniciales a partir de las cuales se lleva a cabo todo el proceso de simulación.

Las condiciones iniciales para el modelo no necesitan gran precisión, a menor precisión de los valores iniciales, mayor tiempo se tomará el modelo para disipar los errores iniciales y converger a la solución correcta. El modelo puede simular flujo permanente a partir de datos aproximados, al final del cual se obtienen los valores correspondientes al estado estacionario, las que a su vez se tomarán como condiciones iniciales para la posterior simulación del flujo no permanente.

\subsection{Condiciones de Frontera Externas}

La solución de las ecuaciones de flujo requiere que se especifiquen condiciones de frontera en los extremos del canal durante todo el tiempo de simulación para proveer el número suficiente de ecuaciones adicionales y satisfacer los requerimientos de la técnica de solución.

Estas condiciones de frontera pueden ser elaboradas a partir de registros históricos o calcularse mediante funciones específicas.

El modelo tiene varias condiciones de frontera externas, estas pueden consistir de una descarga cero (por ejemplo, al final del canal), el caudal o el nivel de la superficie liquida en función del tiempo, o una curva de calibración. También se ha implementado una condición de frontera no reflectante, la cual consiste de una relación matemática que permite que las perturbaciones $\mathrm{u}$ ondas pasen libremente por la frontera y no se reflejen dentro del dominio de cálculo [7].

\subsection{Procedimiento de Solución}

El proceso llevado a cabo en todas las simulaciones es el siguiente: Las condiciones físicas del cauce están reflejadas por las distancias longitudinales y por la batimetría de las secciones transversales. Esto condiciona la variación de los parámetros geométricos de las secciones; es decir, el área, el ancho de la superficie líquida y el perímetro mojado, a partir de los cuales el modelo calcula los parámetros hidráulicos, tales como el radio y el diámetro hidráulico. 
Como ya se ha enunciado, para flujo subcrítico se necesitan dos condiciones de frontera: una para la frontera aguas arriba y otra para la frontera aguas abajo. La condición de frontera aguas arriba está dada por los niveles de la Estación Pucallpillo. Para el proceso de calibración en estado estacionario se tomó solamente el nivel correspondiente al $1^{\circ}$ de marzo de 1983, lo cual determina una condición constante durante todo el proceso de simulación. Para el proceso de verificación en estado no estacionario se tomaron los niveles diarios registrados entre los meses de marzo, abril y mayo de 1983, lo cual determina una condición variable en el tiempo.

La condición de frontera aguas abajo también es diferente para los dos casos. Para el proceso de calibración en estado estacionario se tomó el caudal calculado como condición inicial; es decir el caudal normal, lo que hace una condición constante durante todo el proceso. Para el caso de simulación en flujo no estacionario se tomó como condición de frontera aguas abajo una curva de calibración caudal-nivel. Se sabe que esta curva representa una característica propia de la sección en particular, como en este caso no se tiene esta información en la sección 1-1, se utilizó la curva de calibración que pertenece a la Estación Pucallpillo, la que se ha trasladado a la frontera aguas abajo, asumiendo una relación no exacta.

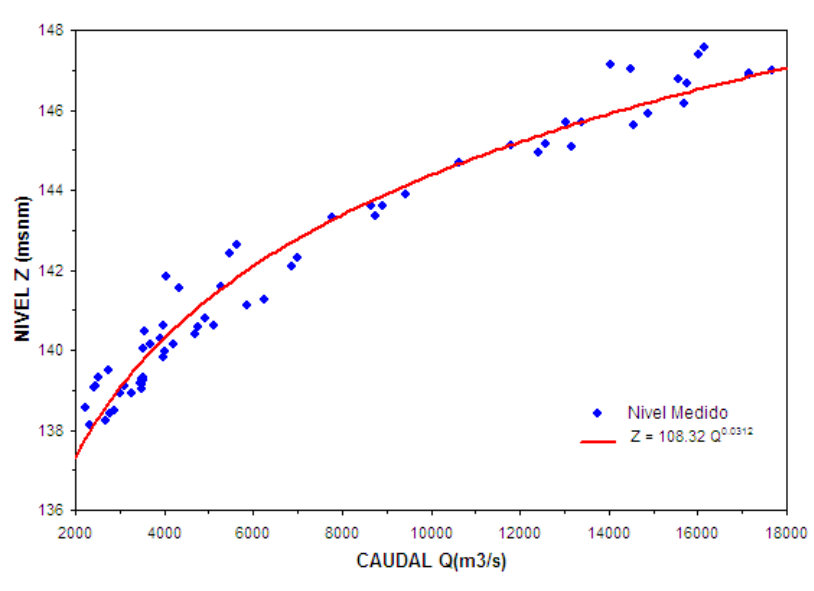

Fig. 9. Curva de calibración de la Estación Pucallpillo

El proceso de calibración consiste en hallar los parámetros físicos y computacionales que reproduzcan las condiciones del flujo para un estado dado. Dicho estado puede ser instantáneo o estacionario, y los resultados se comparan con datos reales históricos o valores medidos en una campaña realizada expresamente para el estudio.

Uno de los parámetros más importantes a ajustar son los coeficientes de rugosidad de Manning, estos valores dependen de muchos factores, entre ellos se encuentra la velocidad del flujo, el nivel del agua, el tipo de lecho y los sedimentos en suspensión [4]. Por lo que prescribir un valor constante para una sección transversal es de hecho una gruesa aproximación.

El proceso de verificación en estado no estacionario sirve como comprobación del modelo; para demostrar que se pueden calcular las variables del flujo en un estado de variación constante. Luego de lo cual se puede proceder a la explotación o utilización del modelo para pronosticar la respuesta del cauce a ciertos cambios físicos, ya sean naturales o artificiales.

\section{Simulación en Estado Estacionario}

Esta prueba consiste en simular flujo estacionario en un canal natural. El tramo tiene una longitud total de $22 \mathrm{kms}$ y una pendiente de fondo $\left(S_{0}\right)$ variable. Los parámetros hidráulicos de las secciones transversales serán interpoladas de la información proporcionada al modelo.

Cada segmento tiene una longitud de 1000 $\mathrm{m}(\Delta x=1000 \mathrm{~m})$, por lo que se tienen 22 segmentos $(n d x=22) \quad$ y $23 \quad$ secciones transversales $(i i=23)$. El tiempo total de simulación es de 24 horas, con un paso de tiempo $(\Delta t)$ de 600 seg., lo que produce 144 pasos de tiempo. $\beta=1.0 \mathrm{yg}=9.81 \mathrm{~m} / \mathrm{s}^{2}$.

Los factores de ponderación del esquema Preissmann tienen los siguientes valores $\theta=0.6, \psi=0.5, \chi=0.5$. Las condiciones iniciales corresponden al caudal normal Qo $=10,918.85 \mathrm{~m}^{3} / \mathrm{s}$ y los niveles de la superficie líquida varían de acuerdo a la tabla 1. Las condiciones de frontera para el tramo son:

\begin{tabular}{|c|c|}
\hline \multicolumn{2}{|c|}{ CONDICIONES DE FRONTERA } \\
\hline Frontera Aguas Arriba & $\begin{array}{c}\mathrm{Z}=145.55 \\
\mathrm{msnm}\end{array}$ \\
\hline Frontera Aguas Abajo & $\begin{array}{c}\mathrm{Q}=10_{3}^{19} / 18.85 \\
\mathrm{~m}^{3} / \mathrm{s}\end{array}$ \\
\hline
\end{tabular}


Para los procesos de calibración y de simulación en estado estacionario se ha considerado que el tramo de estudio tiene dos efluentes y dos tributarios como muestra la figura 5 de la topología utilizada. Esto se hace a fin de representar los caudales que se movilizan a través de los brazos "A" y "B".

Para la simulación en estado estacionario se tienen dos corridas, una considerando que el brazo "A" tiene un caudal constante de $550 \mathrm{~m} 3 / \mathrm{s}$ y el brazo "B" un caudal de 3,100 m3/s y otra corrida considerando que estos caudales se incrementan un 50\% a lo largo del recorrido. Con esto se logra aumentar la velocidad y por tanto reducir los niveles en las secciones aguas abajo de La Hoyada. Esta mejoría es del orden de dos centímetros, lo cual es poco; pero, se presentan estos resultados para resaltar este hecho particular del cauce.
Los coeficientes de rugosidad de Manning ( $n$ ) hallados durante el proceso de calibración son:

Tabla 2. Coeficientes de rugosidad de Manning

\begin{tabular}{|c|c|c|c|}
\hline SECCION & $n$ & SECCION & $n$ \\
\hline 1 & 0.011 & 13 & 0.012 \\
2 & 0.012 & 14 & 0.012 \\
3 & 0.013 & 15 & 0.015 \\
4 & 0.013 & 16 & 0.012 \\
5 & 0.014 & 17 & 0.015 \\
6 & 0.015 & 18 & 0.013 \\
7 & 0.014 & 19 & 0.015 \\
8 & 0.016 & 20 & 0.020 \\
9 & 0.018 & 21 & 0.015 \\
10 & 0.022 & 22 & 0.020 \\
11 & 0.020 & 23 & 0.025 \\
12 & 0.012 & & \\
\hline
\end{tabular}

El perfil longitudinal muestra los resultados luego de las 24 horas de simulación en las 23 secciones transversales. Se observa una pequeña diferencia con los niveles medidos.

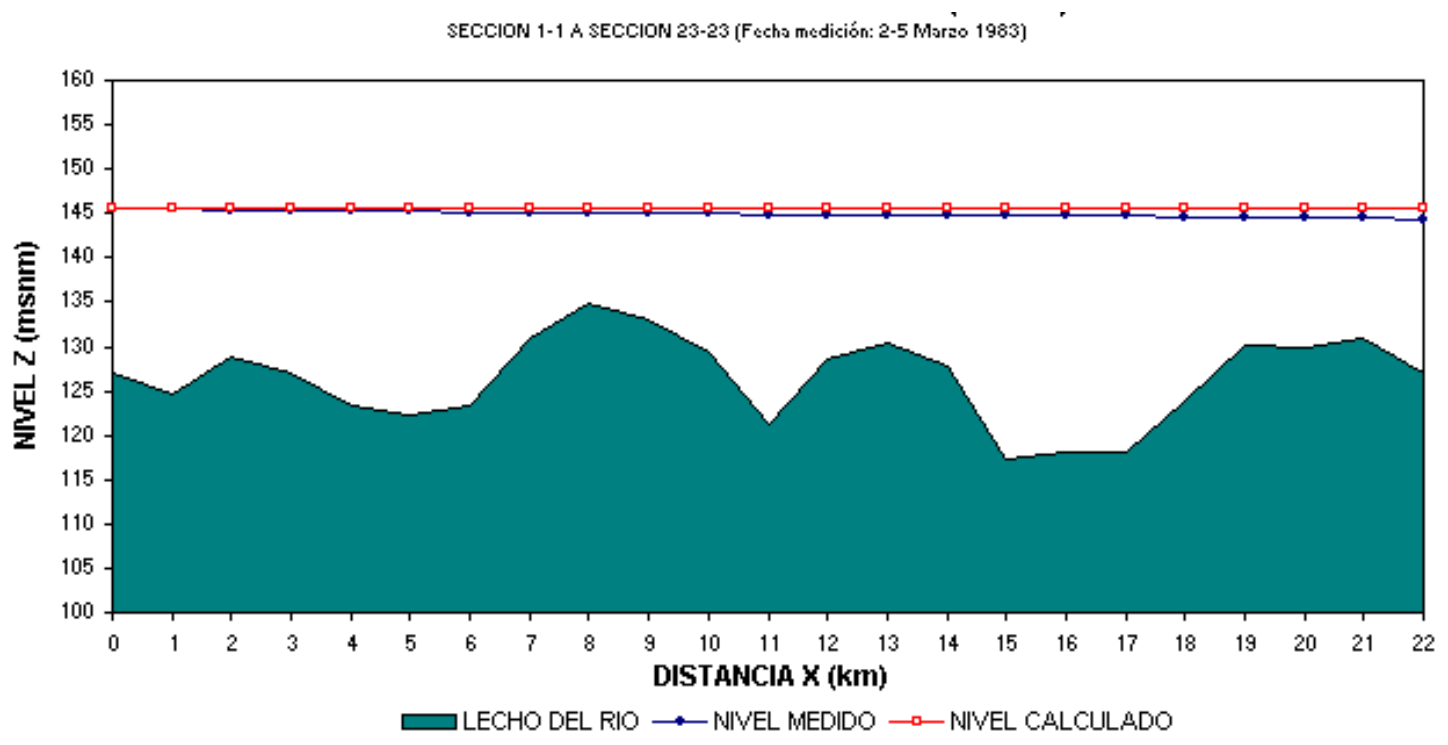

Fig. 10. Perfiles de niveles medidos y calculados - Simulación en estado estacionario

\subsection{Simulación en Estado No Estacionario}

Para el proceso de verificación en estado no estacionario se han tomado las condiciones utilizadas y los coeficientes de Manning de la simulación anterior, solamente se han modificado las condiciones de frontera. El tiempo total de simulación es de 2,208 horas, con un paso de tiempo $(\Delta t)$ de 1,800 seg., lo que produce 4,416 pasos de tiempo.

Para la condición de frontera aguas arriba se han tomados los datos de niveles diarios medidos en la Estación Pucallpillo durante los 
meses de Marzo, Abril y Mayo de 1983.

Para la condición de frontera aguas abajo se utilizó la curva de calibración de la Estación Pucallpillo.

Los resultados de la prueba se han almacenado cada 48 horas de simulación en la estación 10 que corresponde a La Hoyada. Los resultados muestran que la variación de niveles reproduce aceptablemente el flujo no estacionario, aunque se observa una diferencia con los datos registrados, debido principalmente a la condición de frontera utilizada aguas abajo.

\begin{tabular}{|c|c|}
\hline \multicolumn{2}{|c|}{ CONDICIONES DE FRONTERA } \\
\hline Frontera Aguas Arriba & $\mathrm{Z}=\mathrm{Z}(\mathrm{t}) \mathrm{msnm}$ \\
\hline Frontera Aguas Abajo & $\mathrm{Q}=\mathrm{Q}(\mathrm{Z}) \mathrm{m}^{3} / \mathrm{s}$ \\
\hline
\end{tabular}

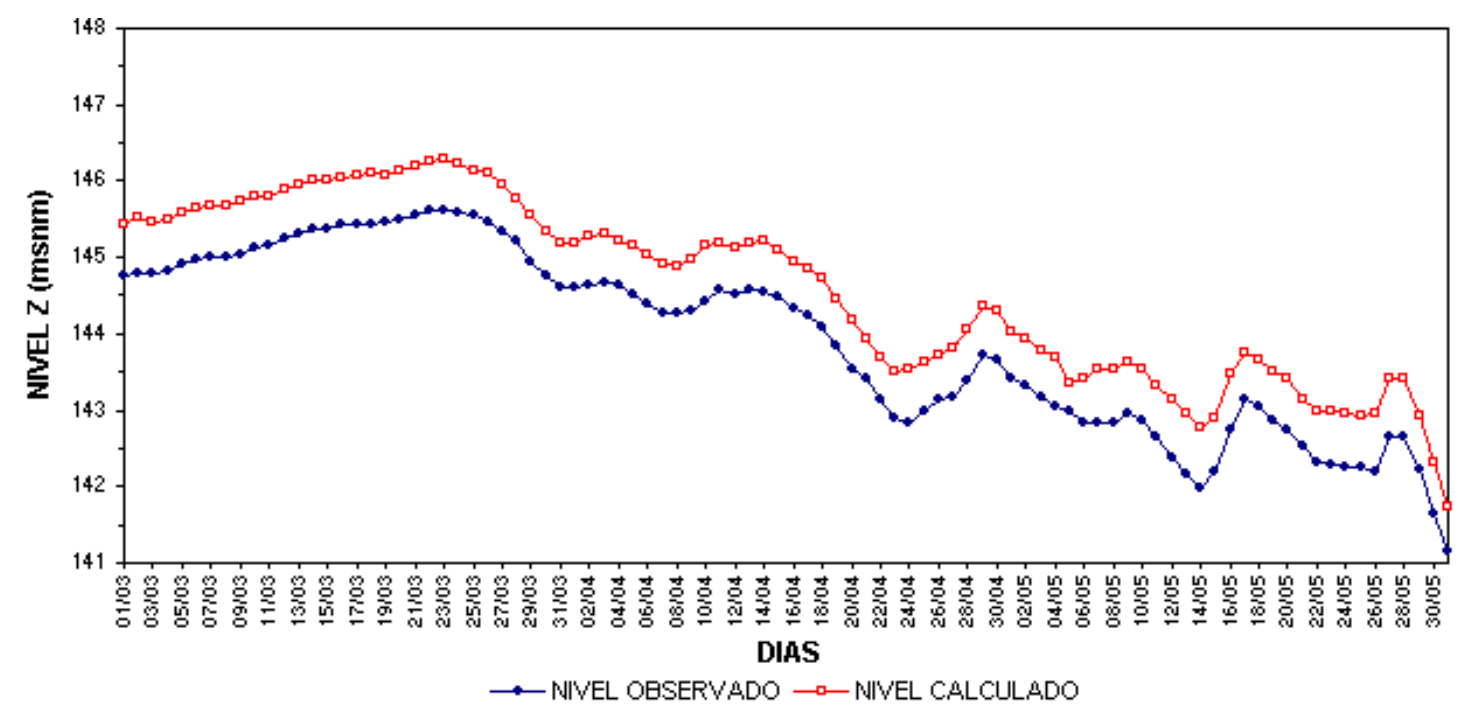

Fig. 11. Histograma de niveles medidos y calculados en la Estación La Hoyada. Simulación en estado no estacionario (Marzo-Mayo 1983)

\section{Conclusiones}

El presente trabajo demuestra que el modelo produce buenos resultados en la simulación del flujo unidimensional en canales naturales. Los resultados de las aplicaciones ilustran la flexibilidad y precisión del modelo para simular diversas condiciones de flujo, tales como flujo uniforme y no uniforme, estacionario y no estacionario.

Las simulaciones realizadas muestran que los registros históricos no se han podido reproducir fielmente, lo cual se ha debido a varios factores. En primer lugar se puede mencionar la falta de información adecuada para el funcionamiento del modelo, no se tienen las mediciones de los niveles de la superficie liquida para todas las estaciones en un mismo instante, sino que tales datos fueron tomados en diferentes días.

También las mediciones batimétricas de todas las secciones transversales no fueron realizadas en un mismo periodo de tiempo; sino que han sido recolectados del 2 al 5 de marzo de 1983, y en este tipo de cauce, debido al enorme volumen de sedimentos transportados, es natural que las condiciones del flujo hayan cambiado en ese lapso de tiempo.

No se tienen histogramas instantáneos ni curvas de calibración en las secciones de control, sobretodo en la frontera aguas abajo. Una curva de calibración sirve solamente para la estación en la cual se ha construido y no se puede 
extrapolar a otra estación. Esto explica la diferencia en los resultados para el caso de la simulación de flujo no estacionario; pero, debido a la carencia de información se tuvo que utilizar dicha curva como condición de frontera aguas abajo.

La técnica de diferencias finitas implícito de Preissmann con sus coeficientes de ponderación confiere un alto grado de flexibilidad para simular diversas condiciones de flujo en canales de propiedades constantes o variables. Además, permite utilizar grandes pasos de tiempo y también discretizar el canal con segmentos de longitudes desiguales.

El modelo también permite especificar valores para el coeficiente de Boussinesq, lo cual es útil para tomar en cuenta las distribuciones de flujo no uniforme que se presentan en las secciones con cambios bruscos de geometría y en los tramos con grandes curvaturas.

Otro aspecto importante es el transporte de sedimentos y la variabilidad del lecho de río. En primer lugar este modelo es hidrodinámico y no contempla el transporte de sedimentos ni los cambios morfológicos del cauce.

El transporte de sedimentos en este río es muy alto y existe una variación volumétrica del lecho muy grande, lo que ocasiona un cambio en las condiciones físicas que no es cuantificable por el modelo.

\section{Referencias}

[1] Abbott M. B., "Computational Hydraulics", Pitman Publishing Limited, London, 1985. 326 pp.

[2] Abbott M. B., Basco D. R., "Computational Fluid Dynamics, An introduction for Engineers", Longman Scientific \& Technical, London, 1989. 425 pp.

[3] Cunge J. A., Holly F. M. and Verwey A., "Practical Aspects of Computational River Hydraulics", Pitman Publishing Limited, London, 1980. $420 \mathrm{pp}$.

[4] Chow V. T., "Open Channel Flow", McGraw Hill Book Company Inc. New York, 1964.

[5] Schaffranek R. W., "Flow Model for Open Channel Reach or Network", U.S. Geological Survey, Professional paper 1384, 1987.

[6] Strelkoff Theodor, "One-dimensional Equations of Open Channel Flow", ASCE, Journal of the Hydraulics Division, v. 95, $\mathrm{N}^{\circ}$ HY3, p. 861-876, 1969.

[7] Vreugdenhil, C. B. Computational Hydraulics. Springer Verlag, Berlin, 1989. 182 pp.

[8] Yzocupe V. A. "Simulación del Tránsito de Avenidas (Flood Routing)", Tesis profesional. UNMSM, EAP de Ingeniería Mecánica de Fluidos. Lima, 1993. 220 pp.

[9] Yzocupe V. A. "Modelamiento Numérico en Ingeniería Hidráulica". Laboratorio de Fluidodinámica Computacional - UNMSM. Lima, 2004. 110 pp. 